\title{
LOCKDOWN: INDIAN DESI RAP'S 'CARTOONIZATION'
}

\section{A study on the inclusion of cartoons in the rap video songs during the Covid-19 lockdown period in India}

\author{
Dr. Yatindra Ingle \\ Usha Pravin Gandhi College of Arts, \\ Commerce and Science
}

Article DOI: https://doi.org/10.36713/epra4853

\begin{abstract}
While the pandemic situation has kept the whole of the nation in a lockdown, this hasn't stopped the content producers to stop their production on alternative media. Likewise, this covd-19 lockdown has engaged the rap video producers into 'cartoonization' of rap videos. This study explores the replacement of real human visuals been replaced by cartoons by the Indian rappers in their videos.
\end{abstract}

KEYWORDS: Rap Music, Cartoonization, alternative media, visuals

\section{INTRODUCTION}

For decades, hip-hop has been perceived as empty and thoughtless, leading to harsh criticism. However, hip-hop has also been effective in creating and expanding the awareness of various social movements, despite being an unorthodox and often overlooked as a successful means. When hip-hop has gained attention, so has a safe-haven for the oppressed 's voice. Unlike other genres, it can speak volumes as it is a staple of youth and popular culture (Ingle, 2020). With the Indian content producers dominating the online spaces for content generation, the alternative media has brought in an ease in making content and publicizing it with zero cost. This has led the alternative media to be a content market without any monopoly on viewership, consumer access, and advertisement their personality and talent. The definition of alternative media should be expressed in relation to material creation, delivery and consumption peculiarities.(Olga G. Bailey, 2007)

Throughout the 1980s, domestic film production expanded to a surge throughout British youth entertainment and music videos from the multimedia culture of New York hip hop. James Macnally explored this historical media transition, the early creation of rap imagery and testimonies of early London hip-hoppers of order to explore how the digital dynamics of music video revealed both the "language of the rank" of hip-hop and new body speeds which facilitated hip Hop transfers as a multifaceted youth movement from New York to London (McNally, 2019)

As the world has nearly stopped due to the lockdown of the Covid-19 pandemic, content producers, on the other hand, are trying to make the best of their productions. On the one hand, where traditional media entertainment industry has been stopped, new media content producers have stepped forward to make the most of this pandemic situation. The situation is that real-life videography is replaced by the use of cartoons in Indian Desi Rap Music Videos. This 'cartoonization' of rap videos is due to the lockdown situation where outdoor videography is banned. Thus, we look at how content producers focused more on visual graphics and animation, giving them a push to produce videos.

The animation can be the simplest and fluid type in the graphic arts in its mature form. Each artist has long searched for the ideal medium-one, which would be colour, light, extend, and motion within his frame, since the first Cro-Magnon Picasso has hacked grafts on his cave wall. It can be done by the illustrated film. There are no constraints of form or length. In technical realism it can approach an absolute and in abstraction it can reach the absolute. You can breathe a deep breath over both. When technically difficult to achieve the unreal or fantastic, the animator does not have any problem (Jones, 1946). The process of its creation, adaptation and decline is also explored. In so doing it shows, along 
with its revolutionary ideas, an important chapter in the little-known early history in electronic synthesized music. (Robertson, 2010). A person can play rap music videos with the music beat of the script by writing text and character gestures (Hayashi, 2017)

A plurality of conclusions is based on the use and fulfilment experience of mass media. Those require an engaged community, an attempt to align media use with gratifications, a variety of accessible contact channels and specific awareness about desires and preferences. "Usually the models of use and gratuitousness begin with social and psychological requirements that encourage certain motivations to select and use forms of communication. Such studies have assumed that a person can choose between communication alternatives knowingly to satisfy the needs if a need leads to a motive. Studies usually takes into account assumptions of the motivations of people to use information channels. The use of this means that compensation or other effects are expected (Rebecca B. Rubin, 1986)

You 're familiar with hip-hop music videos because you've never seen the 'trending' list on YouTube or looked at the famous list of every social media website. Music videos are much more entertaining than any other genres. In this study of genre, it was seen that hip-hop music videos were much more complex than what was thought, and the end product was due to careful planning and many production stages (Sayre, 2019). A systematic analysis of the music video manufacturing industry and the artistic process of rap music video creation and artist promotion is the outcome of this article.
The participants' answers are presented in three areas: the process of music video production, current trends in rap musk videos and the setting of music videos as a gender site. There is no doubt about the artistic freedom of expression and the mechanical production (Fitts, 2007).

Although an artist may have a certain concept, they want their music video entered around, there is usually a large crew that helps create, produce, shoot, edit, and create digital effects for the video (Sayre, Lauren, 2019). This pandemic, brought in the newer concept where no crew, but an individual or a smaller team had been the production team for cartoonization of the rap videos.

\section{METHODOLOGY}

Since the producing of rap video songs involved cartoons leading to a certain approach for audiences, the methodology of content analysis was adopted for this study. Cultivation theory addresses TV's long-term impact on audiences. The theory implies that television's danger lies in its ability to influence not a single point of view on a specific issue, but in its ability to shape the world's moral values and general beliefs. The theory stands on a number of concepts: symbolic environment, storytelling, television symbolic function, television traits, cultural model, value system cultivation, multidirectional process and cultural indicators (Mosharafa, 2015 ). Those rap video songs on produced YouTube's alternative media platform were considered for study.

\section{FINDINGS AND OBSERVATIONS}

\section{Fig.1.1 - A screenshot of the video song "Khatam Karona" produced on youtube with cartoon characters.}

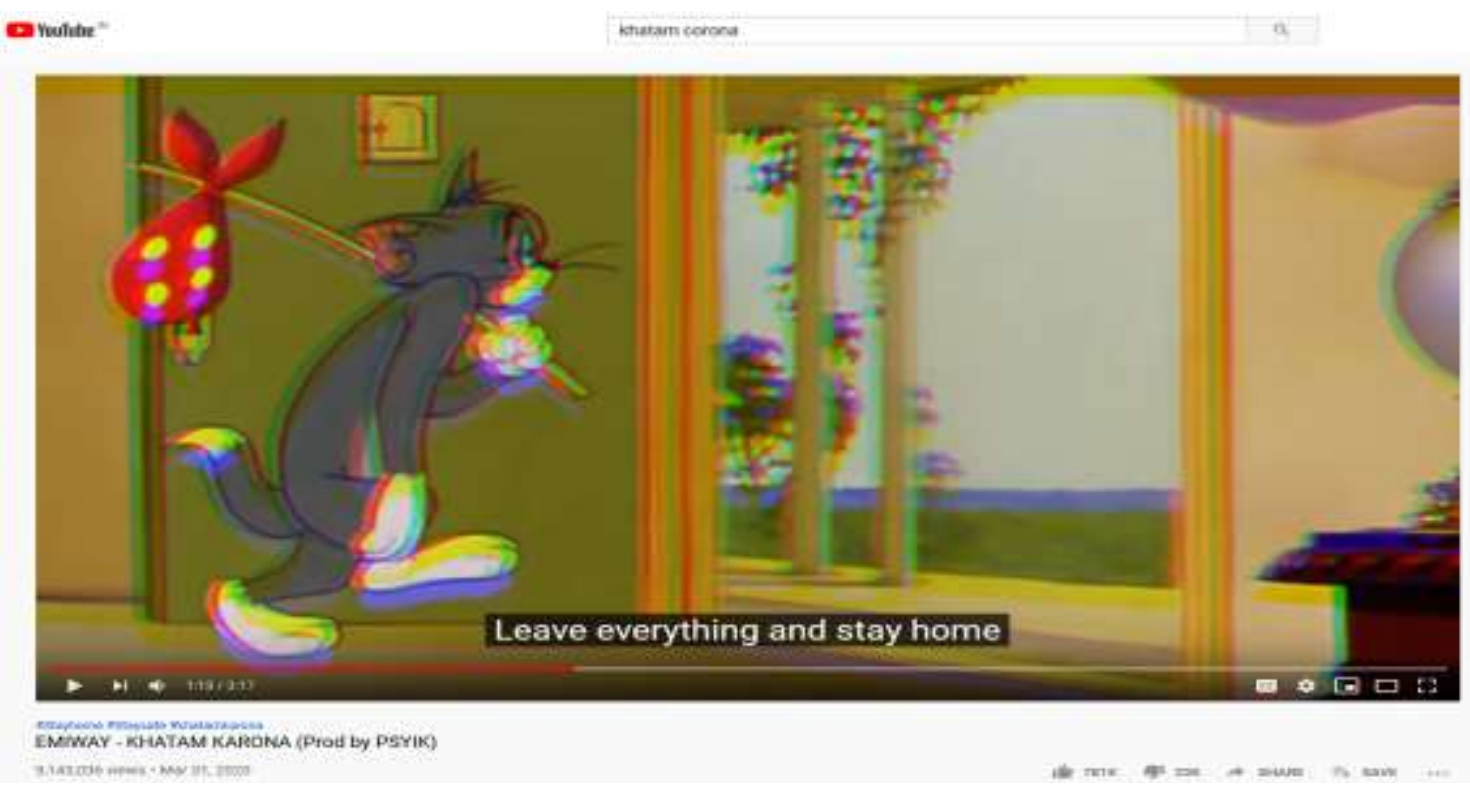


Rappers are American pop culture 's biggest characters. When "natural" shooting doesn't suit a moment 's significance, it's only fair that they receive the animation treatment. Rapper's rap video hit 'Khatam Korona' had a series of animated clips. These clips visualized and narrated corona cause and spread. Such cartoons seemed more appealing when synced with the song's lyric. The video stressed description and lyrics clarification through these cartoons. This rap video seemed more like a video about the corona virus where cartoons were more oriented.

\section{Figure 1.2: A screenshot of the video song "Chaabi Wala Bandar" from YouTube having cartoonization in its visuals.}

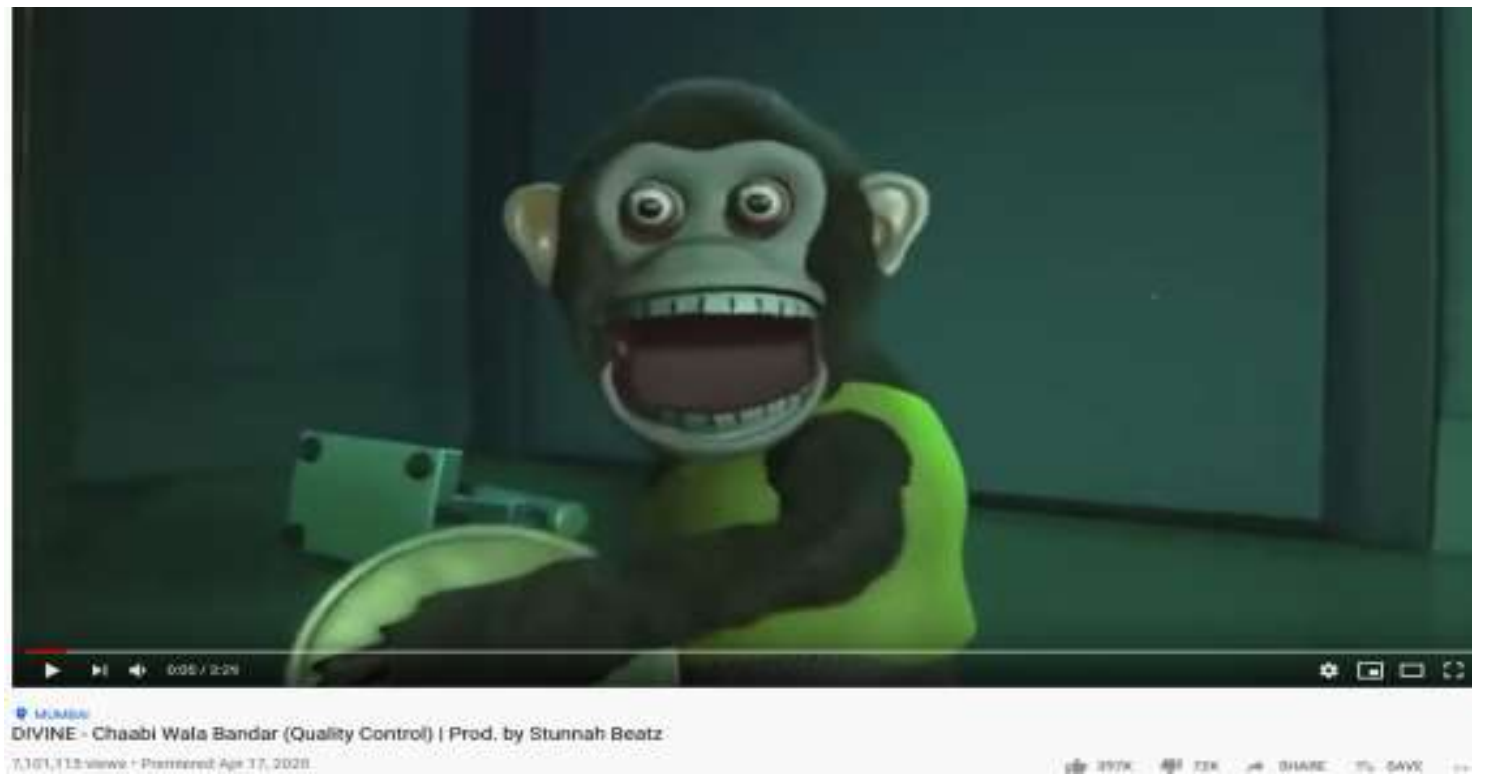

Although Rap songs are primarily part of protest music or music for cause and problems, on the other hand, the rap genre 'Gangsta' brings another dimension of music. Here's a diss (a rap song against another rap artist) album. This track 'Chabiwala Bandar' was also created against another Indian rapper. Like the rap song says, "Chabi" (ring) and "bandar" (moneky) had a monkey toy pounding the drums as the cord coiled. Although this rap song had the rapper in less visual, most monkeys' cartoons were used in this rap. Here, we observed that the reliance on animation and cartoons was important to produce the video during the lockdown era.

Figure 1.3: A screenshot from the rap song "Kuch Bhi Nah" which had Anime characters and was produced on Youtube.

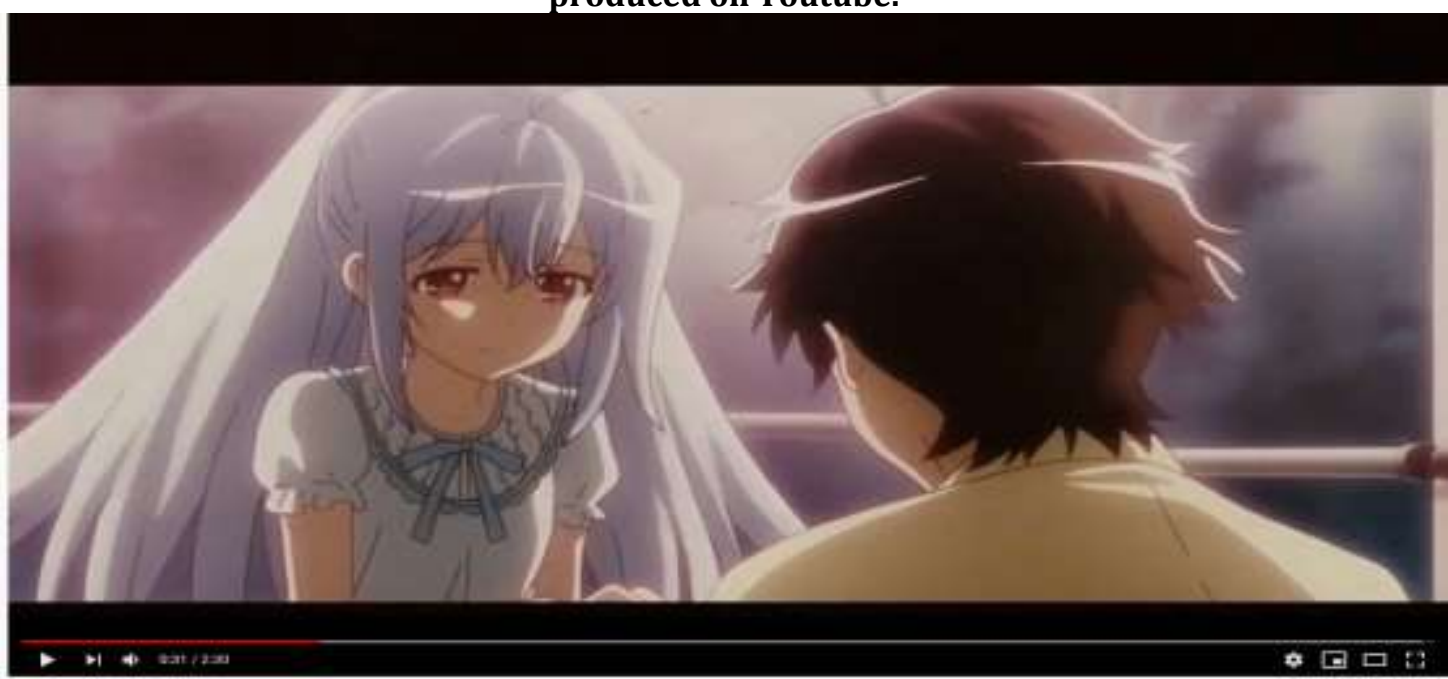

Kuch ahi Nahhi | Animated Video I Proos by Ea I Hindi Rap Sang

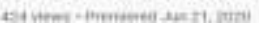


Speaking regarding cartoonization, some rap videos have recorded the use of anime characters. One such rap song was 'Kuch Bhi Nahi' by E3. This rap song is based on a love story characterized by video anime characters. The popularity of anime characters and synchronization with rap and music is another attraction for viewers to like, comment and subscribe to the video song on any alternative media platform,

Figure: 1.4: A screenshot of the rap song "Firse Machayenge" which had the caricatures of the rapper and the $\mathrm{DJ}$ in the video than the real shooting being done.

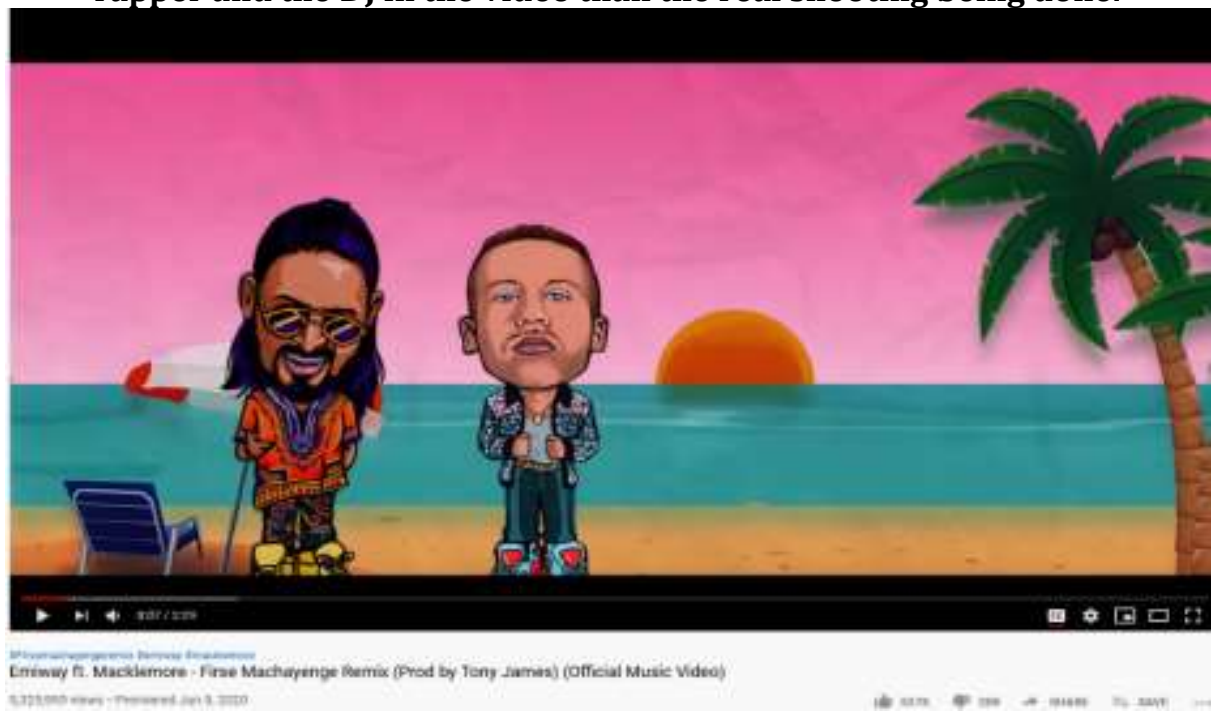

Yet again, the rapper Emiway Buntai remixed and relaunched "Firse Machayenge." Earlier this video had real-life characters, but this remix had the main focus on cartoon-like characterization of rappers. Again, this observation makes us realize the

necessity and indulgence of cartoonization during the pandemic situation.

Figure 1.5: A screen shot from the Rap video song produced by Cartoon Network having the cartoons being animated to follow the tips for not getting sick during the Coivd-19 Pandemic.

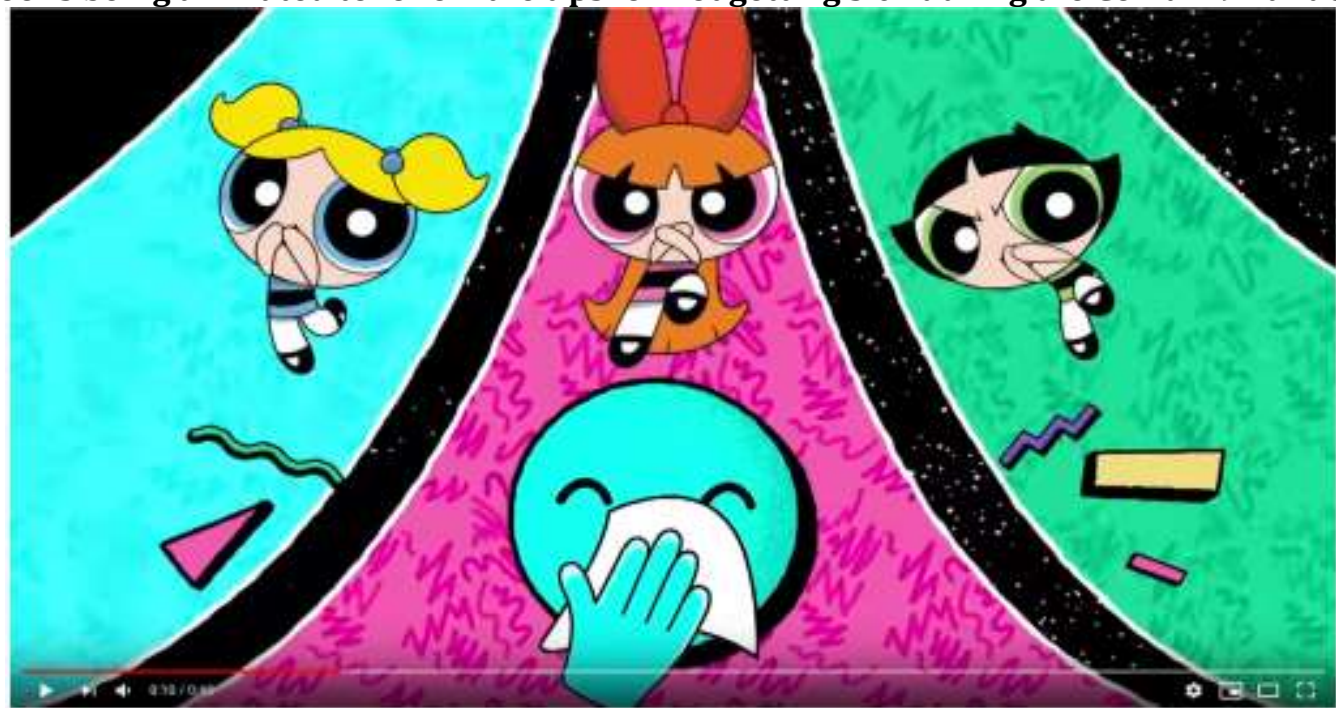

PSA: Tips in Nat Get Sickl! ICanoon Nerwerk

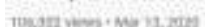

In view of the coronavirus pandemic, Cartoon Network has released a rap song to raise awareness about safety and hygiene. The local music video featuring Ben 10, the Powerpuff Girls and other toon heroes promotes the Be Clean Be Cool message and provides easy helpful tips for children to stay safe and tidy during the current health emergency. Children can learn how simple measures like hand washing, sneezing, and coughing can help prevent disease. 


\section{CONCLUSION}

Although the lockdown period had impeded content producers and videographers from stopping the rap video's real-life filming, they did not disappoint and continued to make the best use of cartoons and animations to be used in rap music videos. Here, producers' reliance on cartoons was observed. While the rappers tried to show themselves in the video, cartoonization filled the need for cartoons and the void. Importantly, it followed a great tradition in hip-hop, where emcees were not afraid to collaborate with animators to create something really vibrant and larger-than - life in this pandemic lockdown situation. Not only cartoons, but the success of cartoons characters has played a key role in that views and shares, as well as video creators or rappers' subscriptions. Here, the rap videos' narrating animation overshadowed more than lyric sync or rap music. The story-telling animation of rap videos, drawn to the cartoons, brought a big aspect of the cartoons becoming part of the rap songs. Furthermore, cartoon animation was such that they clarified rap. Thus, reliance on cartoons and animators brought a new trend among Indian Desi rappers and content producers to introduce the trend of 'cartoonization' in rap videos during India's lockdown.

\section{REFERENCES}

1. Fitts, M. (2007). "Drop It like It's Hot": Culture Industry Laborers and Their Perspectives on Rap Music Video Production. Meridians: feminism, race, $\quad$ transnationalism, 10.2979/mer.2007.8.1.211.

2. Hayashi, M. ,. (2017). Rap music video generator. 2017 IEEE 6th Global Conference on Consumer Electronics, GCCE 2017, (p. 10.1109/GCCE.2017.8229189).

3. Ingle, Y. (2020). Music and Moniker : Promoting Indian National Movement on Social Media through Rap. Our Heritage.

4. Jones, C. (1946). Music and the Animated Cartoon. Hollywood Quarterly Vol. 1, No. 4, 364-370.

5. McNally, J. (2019). Hip-Hop into the Video Age: New York Teenhood, Malcolm McLaren and the British Eye. Visual Culture in Britain, 40-63.

6. Mosharafa, E. (2015 ). All you Need toKnow About: The CultivationTheory. Global Journal of HUMAN-SOCIAL SCIENCE: A Arts \& Humanities - Psychology Volume 15 Issue 8 Version 1.0.

7. Olga G. Bailey, B. C. (2007). Understanding Alternative Media. McGraw-Hill Education .

8. Rebecca B. Rubin, A. M. (1986). Media Use and Meaning of Music Video. Journalism Quarterly, Volume: 63 issue: 2, 353-359.

9. Sayre, L. (2019). Writing Waves: Vol. 1 : Iss. 1 , Article 17. Writing Waves Journal.

10. Sayre, Lauren. (2019). The Art of Hip-Hop in Digital Media. Writing Waves: Vol. 1 : Iss. 1 , Article 17. 